\title{
Synthesis and characterization of iron oxide magnetic nanoparticles
}

\author{
Zbigniew Surowiec, \\ Mieczysław Budzyński, \\ Katarzyna Durak, \\ Grzegorz Czernel
}

\begin{abstract}
Small particles of magnetite, i.e. 7.5, 13.4 and $14.1 \mathrm{~nm}$ in diameter, were obtained by the method of co-precipitation. The crystal structure and size distributions were determined by means of transmission electron microscopy and X-ray diffraction. The magnetic properties of the nanoparticles were tested by Mössbauer spectroscopy within the temperature range from $3 \mathrm{~K}$ to room temperature (RT). The Mössbauer study of magnetic nanoparticles reveals relaxation behaviour related to the existence of the superparamagnetic phase. The blocking temperature depends on the sizes of the nanoparticles and the ammonia concentration.
\end{abstract}

Keywords: Mössbauer effect • superparamagnetic nanoparticles

\section{Z. Surowiec ${ }^{\bowtie}$, M. Budzyński, K. Durak}

Institute of Physics,

Maria Curie-Skłodowska University,

1 M. Curie-Skłodowskiej Sq., 20-031 Lublin, Poland,

Tel.: +48 81537 6220, Fax: +48 815376191 ,

E-mail: zbigniew.surowiec@umcs.lublin.pl

\section{G. Czernel}

Department of Biophysics,

University of Life Sciences in Lublin,

13 Akademicka Str., 20-001 Lublin, Poland

Received: 15 July 2016

Accepted: 27 November 2016

\section{Introduction}

Magnetic nanoparticles (MNPs) have drawn much attention because they can be widely used in electronics [1], nanotechnology [2] and most of all in medicine [3-5]. In external magnetic fields they can be useful as contrast agents for magnetic resonance imaging (MRI) [6, 7], as colloidal mediators for the treatment of cancer using magnetic hyperthermia $[8,9]$ or as intelligent drug delivery systems [6]. In medical applications, the most common MNPs are iron oxides, especially magnetite and maghemite. These oxides receive much attention because of their following properties: biocompatibility, low toxicity and antimicrobial effectiveness [10].

Stabilization of the surface coating of iron oxide nanoparticles is an important issue because these particles tend to aggregate when dispersed into solvents or biological media. The high surface area to volume ratio of nanoparticles usually results in high rates of reactivity and colloidal instability compared to their bulk counterparts. Nanoparticles exhibit a tendency to agglomerate in order to reduce their surface energy by strong magnetic dipole-dipole interactions between particles. Hence, one of the main problems of producing a stable magnetic fluid is preventing agglomeration during the synthesis and coating processes. Coatings used for iron oxide nanoparticles can consist of various types of molecules, for example inorganic molecules, small organic molecules, polymers and proteins [11-14].

Magnetic particles with a size in the order of several nanometres may exhibit superparamagnetic properties. In these sufficiently small nanoparticles, the direction of magnetization can randomly invert 
as a result of fluctuations in temperature. The typical time interval between two orientation inversions is given by the Néel-Arrhenius equation

$$
\tau_{\mathrm{N}}=\tau_{0} \exp \left(\frac{K V}{k_{\mathrm{B}} T}\right)
$$

where $K$ is the magnetic anisotropy energy density of the particle, $V$ is the volume of the particle, $k_{\mathrm{B}}$ is the Boltzmann constant and $T$ is the temperature. $\tau_{0}$ is the attempt time with a value of between $10^{-9}$ and $10^{-10} \mathrm{~s}$. The superparamagnetic relaxation of magnetite nanoparticles depends on the volume of the particle and the temperature. On the other hand, if magnetic interactions between the particles are not negligible, they can significantly influence superparamagnetic relaxation.

The aim of the present study is to synthesize surfactant-coated magnetite nanoparticles by the method of co-precipitation, to investigate the influence of the ammonia concentration on the size of magnetite nanoparticles prepared in homogeneous aqueous media as well as to characterize their magnetic properties using Mössbauer spectroscopy.

\section{Experimental}

Nanoparticles were obtained by co-precipitation at room temperature. In the preparation process, the following pure chemicals were used: $\mathrm{FeCl}_{2} \cdot 4 \mathrm{H}_{2} \mathrm{O}$ (Aldrich, 99\%), $\mathrm{FeCl}_{3} \cdot 6 \mathrm{H}_{2} \mathrm{O}$ (Aldrich, 98\%), 56\% $\mathrm{NH}_{4} \mathrm{OH}$ with $25 \% \mathrm{NH}_{3}$, DMSA $\mathrm{C}_{4} \mathrm{H}_{6} \mathrm{O}_{4} \mathrm{~S}_{2}$ (Aldrich, 98\%) and distilled water. During the synthesis, the solutions were mixed by a magnetic stirrer. Three samples were made as follows:

- sample 1 was prepared by dissolving appropriate amounts of iron II $\left(\mathrm{FeCl}_{2} \cdot 4 \mathrm{H}_{2} \mathrm{O}\right)$ and III $\left(\mathrm{FeCl}_{3} \cdot 6 \mathrm{H}_{2} \mathrm{O}\right)$ in distilled water in two separate test tubes. Next, the contents of both test tubes were mixed and aqueous ammonia $\left(56 \% \mathrm{NH}_{4} \mathrm{OH}\right.$ with $25 \% \mathrm{NH}_{3}$ ) was added dropwise;

- in the cases of samples 2 and 3, an aqueous solution of iron II and III was added dropwise to the solution of ammonia.

The ammonia concentration in samples 1 and 2 was $1.5 \mathrm{M}$ and in the third sample was $14 \mathrm{M}$.

The weighed amounts of iron II and III in each sample were the same. The dark sediments obtained were washed with four $30 \mathrm{ml}$ batches of distilled water. A permanent magnet was used to separate the nanoparticles from the solution. The method of magnetically controlling the nanoparticles using the permanent magnet is shown in Fig. 1. In the next step, the three samples were coated with dimercaptosuccinic acid (DMSA). First, a $2.7 \times 10^{-3} \mathrm{M}$ solution of DMSA was prepared. Next, $10 \mathrm{ml}$ of water was added to the suspension of nanoparticles and stirred under a nitrogen atmosphere. The final step was the admixing of an appropriate amount of ferrofluid to $1.5 \mathrm{ml}$ of DMSA. The magnetic nanoparticles prepared in this way were studied using Mössbauer spectroscopy (MS) and X-ray diffraction (XRD). Additionally, analysis of the obtained transmission electron microscope (TEM) images was performed.

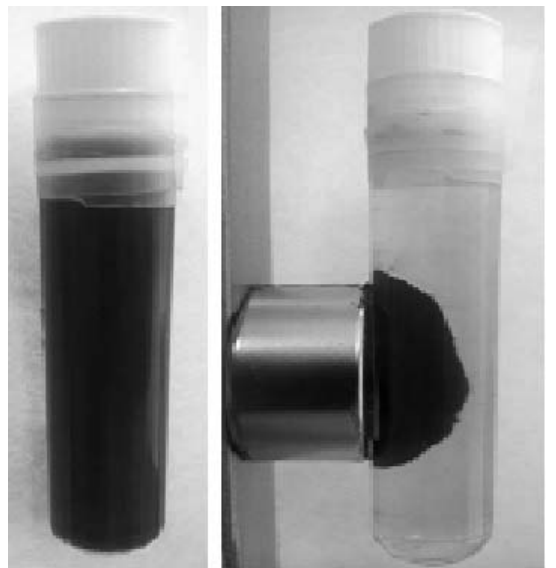

Fig. 1. Magnetic separation of superparamagnetic nanoparticles dispersed in liquid media. The separation-redispersion cycles can be repeated indefinitely. Originally suspended (left) were attracted to the side of a permanent magnet (right) after $5 \mathrm{~min}$.

XRD patterns were measured by means of a Philips PANalytical X'Pert PRO PW 3040/60 X-ray diffractometer using $\mathrm{CuK} \alpha$ radiation at room temperature. They were fitted using the PANalytical X'Pert HighScore Plus program for Rietveld refinement.

The Mössbauer spectra of powdered samples were recorded in transmission geometry at different temperatures between $3 \mathrm{~K}$ and room temperature (RT) using a constant acceleration spectrometer with a ${ }^{57} \mathrm{Co} / \mathrm{Rh}$ source.

\section{Results}

The X-ray diffraction measurements were carried out to determine the crystal structure of the particles. The XRD patterns obtained for the three samples are presented in Fig. 2. The shape and position of

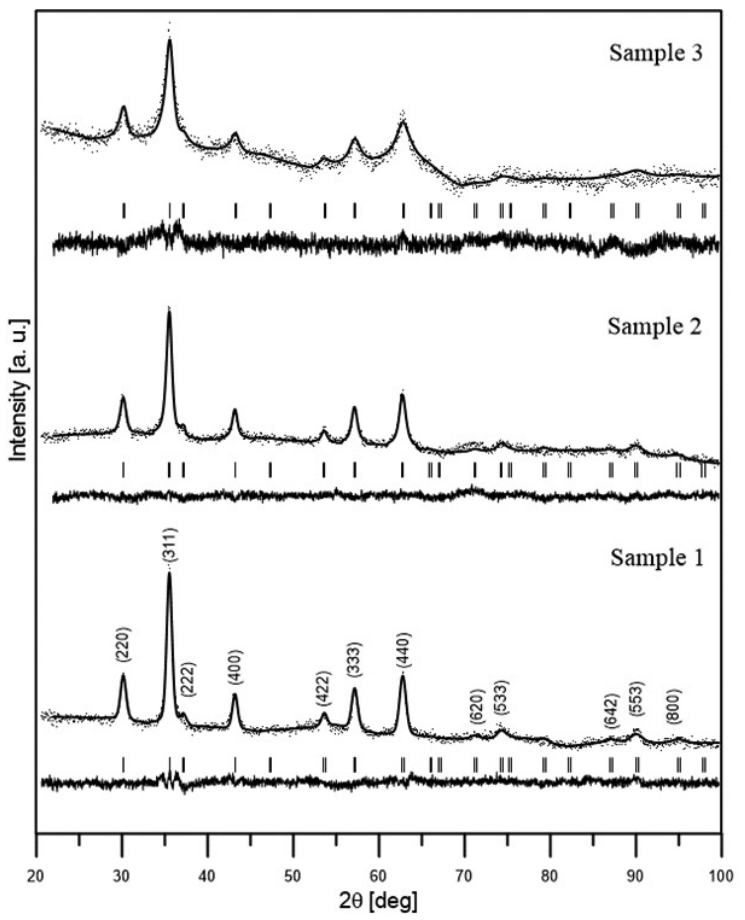

Fig. 2. The XRD diffraction patterns of $\mathrm{Fe}_{3} \mathrm{O}_{4}$ for samples $1-3$. 
the main diffraction peaks are characteristic of the cubic spinel structure. The absence of additional reflections in the diffraction pattern confirms the purity of the phase. The sizes of the nanoparticles and lattice strains were determined from the diffraction peak broadening using the Williamson-Hall technique [15]. The mean crystallite sizes of samples 1 and 2 are similar, $14.1 \mathrm{~nm}$ and $13.4 \mathrm{~nm}$, respectively. In sample 3, which was prepared at the highest ammonia concentration, the mean diameter of magnetite nanoparticles was equal to $7.5 \mathrm{~nm}$. The values of the crystallite sizes, microstrain parameters and lattice constants are presented in Table 1 . The lattice constants are the greatest for the smallest nanoparticles because their surface area to volume ratio is the highest.

The particle size distribution and morphology were examined by transmission electron microscopy (TEM). As shown in Fig. 3 the magnetite nanoparticles in sample 3 are well separated, though their shape is somewhat irregular. The $\mathrm{Fe}_{3} \mathrm{O}_{4}$ nanoparticle size distribution obtained from the analysis of the TEM images is presented in Fig. 4. The parameters of the particle size distribution were calculated using the following equation based on a lognormal distribution function [16].

$$
P(d)=\frac{1}{x \sigma_{d} \sqrt{2 \pi}} \exp \left(-\frac{d^{2}}{2 \sigma_{d}^{2}}\right)
$$

where $d=\ln \left(x / x_{0}\right), \sigma_{d}$ is the standard deviation of $d$ and $x_{0}$ is the mean particle diameter.

Table 1. The crystal structure parameters of magnetite nanoparticles at various ammonia concentrations : a - lattice constant, $\eta$ - lattice strain, $d$ - nanoparticle diameter

\begin{tabular}{lcccc}
\hline & $\begin{array}{c}\text { Ammonia } \\
\text { concentration }\end{array}$ & $\begin{array}{c}\mathrm{a} \\
{[\AA]}\end{array}$ & $\begin{array}{c}\eta \\
{[\%]}\end{array}$ & $\begin{array}{c}d \\
{[\mathrm{~nm}]}\end{array}$ \\
\hline Sample 1 & $1.5 \mathrm{M}$ & $8.367(2)$ & 0.23 & 14.1 \\
Sample 2 & $1.5 \mathrm{M}$ & $8.367(2)$ & 0.28 & 13.4 \\
Sample 3 & $14 \mathrm{M}$ & $8.335(2)$ & 1.12 & 7.5 \\
\hline
\end{tabular}

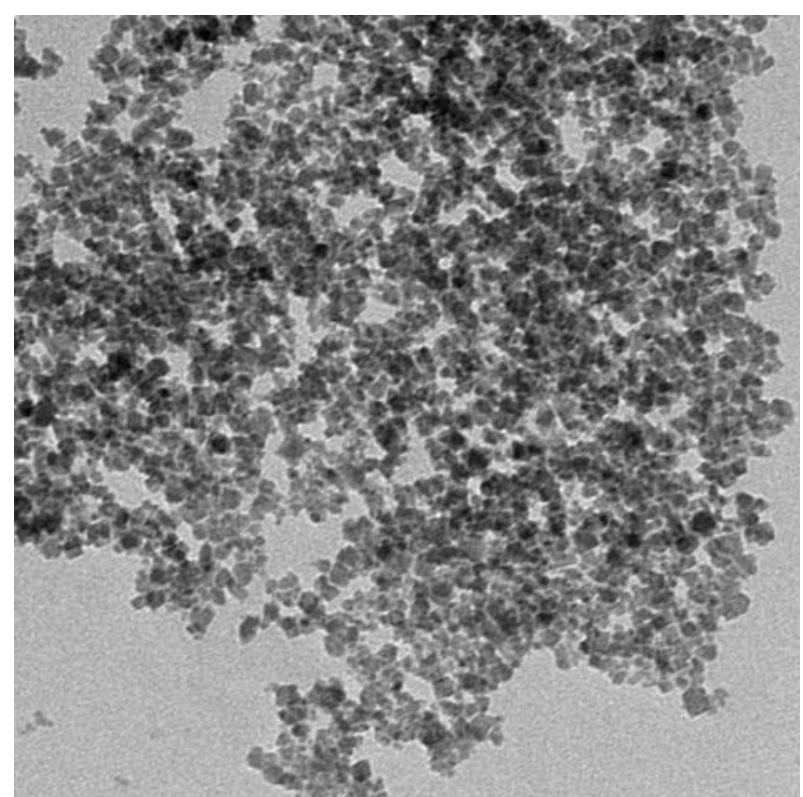

Fig. 3. TEM image of DMSA-coated magnetite nanoparticles in sample 3 .

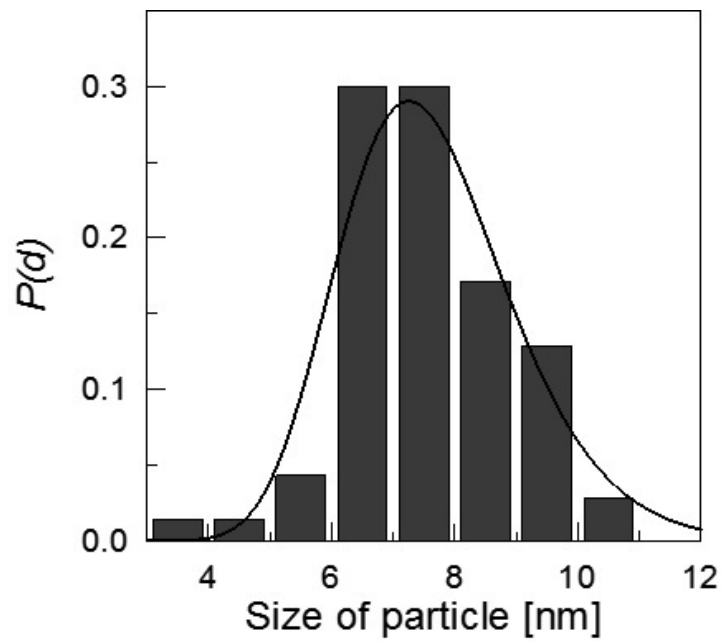

Fig. 4. Particle size distribution determined from TEM images of sample 3 .

The mean particle diameter $x_{0}$ determined in this way is $7.51 \mathrm{~nm}$ with a standard deviation $\sigma_{d}$ of 0.18 which is exactly the same value as the mean particle diameter calculated from the XRD data for sample 3.

Figure 5a shows the Mössbauer spectrum recorded at RT for sample 1 . The spectrum was fitted with three components. Two sextets correspond to the octahedral (A) and tetrahedral (B) Fe positions in the magnetite structure. In stoichiometric bulk magnetite the ratio between the intensity of sextets $A$ and $B$ is 1:2. However, this ratio can be different for nanoparticles and it can depend on the synthesis conditions such as temperature, time, $\mathrm{pH}$ and the kind of dissolvent $[17,18]$. The last sextet that corresponds to the lowest magnetic field exhibits strongly broadened lines. This can be associated with the surface disorder and magnetic interactions between the nanoparticles [19]. Although the diameter of the nanoparticles is equal to $14 \mathrm{~nm}$, superparamagnetic relaxation is absent at RT.

Figure $5 \mathrm{~b}$ shows the Mössbauer spectra recorded at different temperatures for sample 2 . The spectrum obtained at RT is similar to that obtained for sample 1 but an additional doublet is observed in the central part of the spectrum. This doublet component is related to the superparamagnetic relaxation of the nanoparticles. As the temperature decreases the contribution of the doublet decreases. Below $70 \mathrm{~K}$ the superparamagnetic oscillations disappear.

The Mössbauer spectra collected for sample 3 (with a mean magnetite nanoparticle diameter of $7.5 \mathrm{~nm}$ ) at different temperatures are shown in Fig. 5c. It can be seen that only a doublet is recorded at RT. When the temperature decreases below RT a sextet is observed. The superparamagnetic doublet disappears when the temperature decreases below $50 \mathrm{~K}$.

The characteristic measurement time in milliseconds, $\tau_{1}$, corresponds to the Larmor precession period of the ${ }^{57} \mathrm{Fe}$ nucleus. For fine nanoparticles the relaxation time, $\tau$, is smaller than the $\tau_{1}$, and the magnetic fluctuation is so fast that the average internal magnetic hyperfine field at the ${ }^{57} \mathrm{Fe}$ nuclei is equal to zero. In this case a doublet can be observed in the 


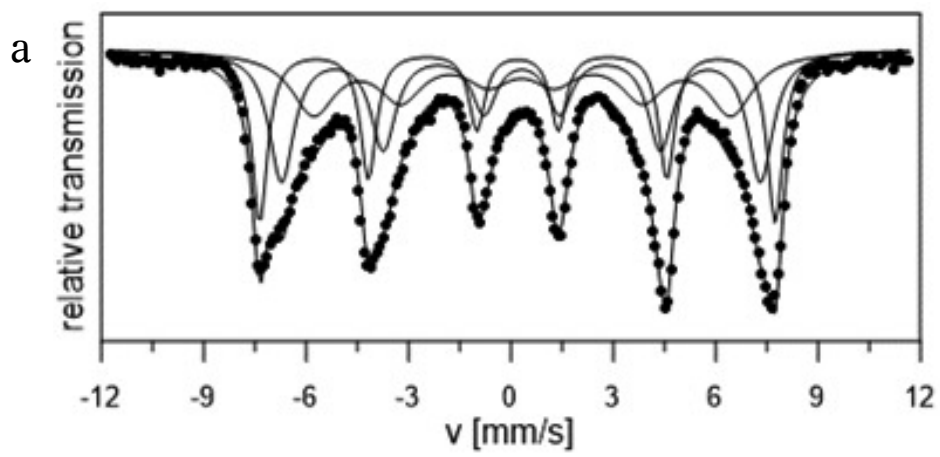

b

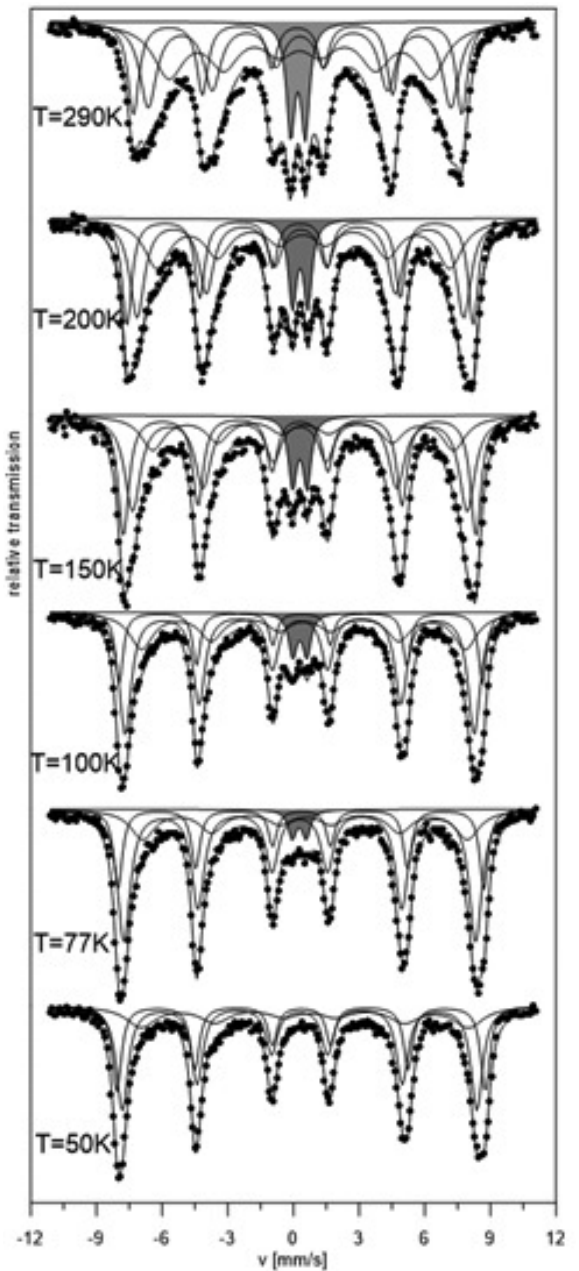

c

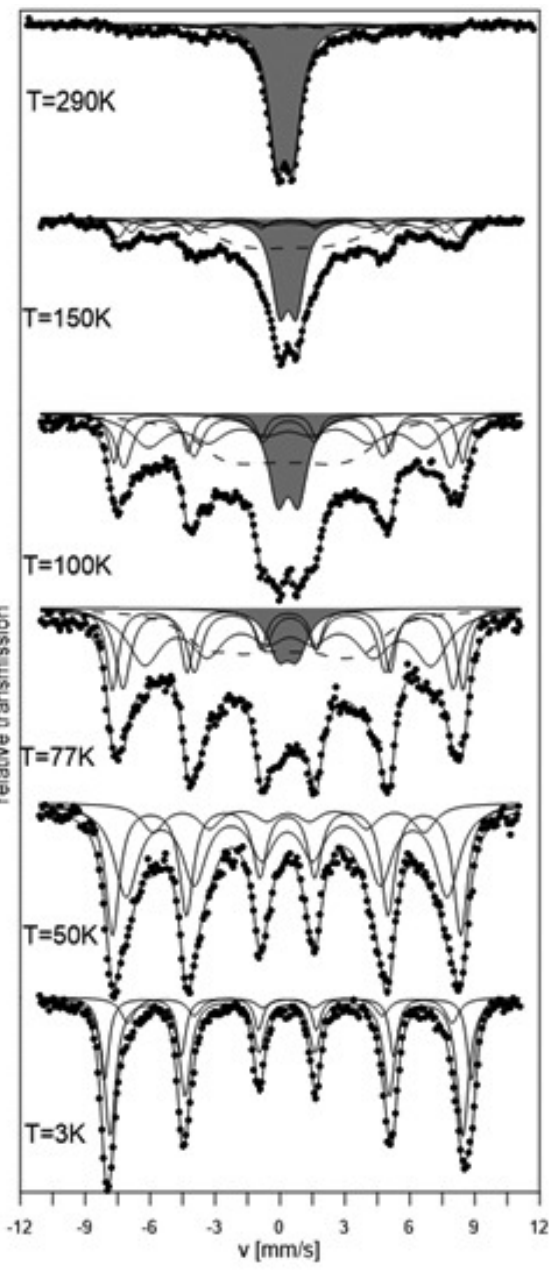

Fig. 5. ${ }^{57} \mathrm{Fe}$ Mössbauer spectra of magnetite nanoparticles for sample 1 obtained at RT (a). ${ }^{57} \mathrm{Fe}$ Mössbauer spectra of magnetite nanoparticles for sample 2 (b) and for sample 3 (c) obtained at different temperatures.

Mössbauer spectrum. As the temperature decreases $\tau$ increases and can become equal to $\tau_{1}$. If this occurs, some ${ }^{57} \mathrm{Fe}$ nuclei undergo rapid superparamagnetic relaxation and a doublet and sextet coexist in the pattern. As temperature decreases the relaxation of nanoparticles becomes much slower and the spectrum takes on the form of a sextet.

\section{Conclusions}

The magnetite nanoparticles $\mathrm{Fe}_{3} \mathrm{O}_{4}$ were obtained by the method of co-precipitation. They were coated with a DMSA surfactant in order to prevent aggregation.
The fixed ammonia concentration does not influence the size of the nanoparticles during this method of synthesis but it has a significant impact on magnetic properties. In the sample obtained by adding an aqueous solution of ammonia dropwise to the solution of iron II and III, the superparamagnetic oscillations were not observed at room temperature, while the sample obtained by adding iron ions dropwise in the aqueous ammonia solution exhibited a significant superparamagnetic component. On the other hand, it was observed that the size of the nanoparticles depends on the ammonia concentration during the preparation process. As the ammonia concentration increases, the size of nanoparticles decreases. It can be concluded that the magnetic 
properties of superparamagnetic nanoparticles depend on their size and the method of synthesis.

Synthesized nanoparticles show superparamagnetic fluctuations, which are advantageous in hyperthermia or MRI. The results obtained can provide a better understanding of the influence of pre-treatment conditions on the magnetic properties of the coated magnetite nanoparticles.

\section{References}

1. Xu, S., Habib, A. H., Pickel, A. D., \& McHenry, M. E. (2015). Magnetic nanoparticle-based solder composites for electronic packaging applications. Prog. Mater. Sci., 67, 95-160. http://dx.doi.org/10.1016/j. pmatsci.2014.08.001.

2. Zahn, M. (2001). Magnetic fluid and nanoparticle applications to nanotechnology. J. Nanopart. Res., 3, 73-78

3. Tartaj, P., Puerto Morales, M., Veintemillas-Verdaguer, S., Gonzalez-Carreno, T., \& Serna, C. J. (2003). The preparation of magnetic nanoparticles for applications in biomedicine. J. Phys. D-Appl. Phys., 36, R182-R197. stacks.iop.org/JPhysD/36/R182.

4. Duguet, E., Vasseur, S., Mornet, S., \& Devoisselle, J. M. (2006). Magnetic nanoparticles and their applications in medicine. Nanomedicine, 1, 157-168. DOI: 10.2217/17435889.1.2.157.

5. Ito, A., Shinkai, M., Honda, H., \& Kobayashi, T. (2005). Medical application of functionalized magnetic nanoparticles. J. Biosci. Bioeng., 100, 1-11. DOI: $10.1263 /$ jbb.100.1.

6. Sun, C., Lee, J. S. H., \& Zhang, M. (2008). Magnetic nanoparticles in MR imaging and drug delivery. $A d v$. Drug Deliv. Rev., 60, 1252-1265. DOI: 10.1016/j. addr.2008.03.018.

7. Veiseh, O., Gunn, J. W., \& Zhang, M. (2010). Design and fabrication of magnetic nanoparticles for targeted drug delivery and imaging. Adv. Drug Deliv. Rev., 62, 284-304. DOI: 10.1016/j.addr.2009.11.002.

8. Lacroix, L. M., Bel Malaki, R., Carrey, J., Lachaize, S., Respaud, M., Goya, G. F., \& Chaudret, B. (2009). Magnetic hyperthermia in single-domain monodisperse FeCo nanoparticles: Evidences for StonerWohlfarth behavior and large losses. J. Appl. Phys., 105, 023911. DOI: 10.1063/1.3068195.

9. Subramanian, M., Miaskowski, A., Pearcec, G., \& Dobsond, J. (2015). A coil system for realtime magnetic fluid hyperthermia microscopy studies. Int. J. Hyperther., 32, 112-120. DOI: 10.3109/02656736.2015.1104732.

10. Wu, W., He, Q., \& Jiang, C. (2008). Magnetic iron oxide nanoparticles: Synthesis and surface functionalization strategies. Nanoscale Res. Lett., 3, 397-415. DOI: 10.1007/s11671-008-9174-9.

11. Bumb, A., Brechbiel, M. W., Choyke, P. L., Fugger, L., Eggeman, A., Prabhakaran, D., Hutchinson, J., \& Dobson, P. J. (2008). Synthesis and characterization of ultra-small superparamagnetic iron oxide nanoparticles thinly coated with silica. Nanotechnology, 19 335601. DOI: 10.1088/0957-4484/19/33/335601.

12. Taupitz, M., Wagner, S., Schnorr, J., Kravec, I., Pilgrimm, H., Bergmann-Fritsch, H., \& Hamm, B. (2004). Phase I clinical evaluation of citrate-coated monocrystalline very small superparamagnetic iron oxide particles as a new contrast medium for magnetic resonance imaging. Invest. Radiol., 39, 394-405.

13. Zhang, M. Q., Zhang, Y., \& Kohler, N. (2002). Surface modification of superparamagnetic magnetite nanoparticles and their intracellular uptake. Biomaterials, 23, 1553-1561. DOI: 10.1016/S01429612(01)00267-8.

14. Wiogo, H. T. R., Lim, M., Bulmus, V., Yun, J., \& Amal, R. (2011). Stabilization of magnetic iron oxide nanoparticles in biological media by fetal bovine serum (FBS). Langmuir, 27, 843-850. DOI: 10.1021/ La104278m.

15. Williamson, G. K., \& Hall, W. H. (1952). X-ray line broadening from filed aluminium and wolfram. Acta Metall., 1, 22-31. DOI: 10.1016/00016160(53)90006-6.

16. Jeong, J. R., Shin, S. C., Lee, S. J., \& Kim, J. D. (2005) Magnetic properties of superparamagnetic $\gamma-\mathrm{Fe}_{2} \mathrm{O}_{3}$ nanoparticles prepared by coprecipitation technique. J. Magn. Magn. Mater., 286, 5-9. DOI: 10.1016/j. jmmm.200.09.129.

17. Kalska-Szostko, B., Zubowska, M., \& Satuła, D. (2006). Studies of the magnetite nanoparticles by means of Mössbauer spectroscopy. Acta Phys. Pol. A, 109, 365-369.

18. Marín, T., Montoya, P., Arnache, O., \& Calderón, J. (2016). Influence of surface treatment on magnetic properties of $\mathrm{Fe}_{3} \mathrm{O}_{4}$ nanoparticles synthesized by electrochemical method. J. Phys. Chem. B, 120, 6634-6645. DOI: 10.1021/acs.jpcb.6b01796.

19. Mørup, S., Hansen, M. F., \& Frandsen, C. (2010). Magnetic interactions between nanoparticles. Beilstein J. Nanotechnol., 1, 182-190. DOI: 10.3762/ bjnano.1.22. 